\title{
Eficiência de trifloxistrobina e tebuconazole, em formulação comercial, associados a fungicidas protetores no controle da mancha marrom de Alternária em tangor "Murcott"
}

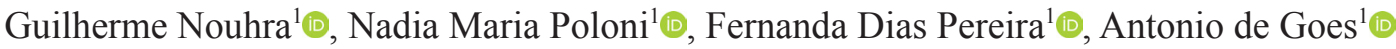

\begin{abstract}
${ }^{1}$ UNESP/FCAV - Universidade Estadual Paulista "Júlio de Mesquita Filho" - Faculdade de Ciências Agrárias e Veterinárias - Câmpus de Jaboticabal, Via de Acesso Prof. Paulo Donato Castellane, s/n - CEP 14884-900, Jaboticabal, SP, Brasil.

Autor para correspondência: Nadia Poloni (nadiapoloni@gmail.com)

Data de chegada: 09/11/2020. Aceito para publicação em: 26/03/2021.
\end{abstract}

$10.1590 / 0100-5405 / 245415$

\section{RESUMO}

Nouhra, G.; Poloni, N.M.; Pereira, F.D.; Goes, A. Eficiência de trifloxistrobina e tebuconazole, em formulação comercial, associados a fungicidas protetores no controle da mancha marrom de Alternária em tangor "Murcott”. Summa Phytopathologica, v.47, n.2, p.122-125, 2021.

\begin{abstract}
Dentre as doenças fúngicas, a mancha marrom de Alternaria (MMA) é a doença mais importante para tangor "Murcott", causando desfolha, mancha e queda de frutos e, com isso, acarretando muitos prejuízos. Nesse trabalho foi avaliada a eficiência de trifloxistrobina e tebuconazol, em formulação comercial, combinados com pirimetanil, em duas dosagens, e combinado com cobre solúvel em água, comparado com oxicloreto de cobre, no controle da MMA em pomar de tangor 'Murcott', com 15 anos de plantio, em Estiva Gerbi/SP. Os tratamentos foram constituídos pela mistura de tanque de trifloxistrobina e tebuconazole a $0,80 \mathrm{~L} \mathrm{ha}^{-1}$ com os seguintes fungicidas (L ou kg de produto comercial 2000 $\left.\mathrm{L}^{-1}\right)$ : T1- pirimetanil $(1,0)$; T2- pirimetanil $(1,5)$; T3- sulfato de cobre solúvel $(1,0)$; T4- oxicloreto de cobre $(3,60)$. Foi adotado o delineamento de Split-
\end{abstract}

block, em subparcelas, com três repetições de dez plantas na linha entre as duas linhas adjacentes. Os fungicidas foram aplicados mediante turbopulverizador FM Copling, sendo empregada uma vazão de $100 \mathrm{~mL}\left(\mathrm{~m}^{3}\right)^{-1}$. No total foram realizadas 17 aplicações de fungicidas, em intervalos que variaram de 12 a 15 dias. As avaliações consistiram na determinação dos níveis de severidade dos sintomas nos frutos, determinados em três épocas (S1, S2 e S3) a determinação do número de cicatrizes nos frutos, quando realizada a colheita. Verificou-se que os tratamentos constituídos pelas combinações de trifloxistrobina e tebuconazole e pirimetanil, em duas dosagens, foram eficientes no controle do patógeno, assemelhando-se aos resultados do tratamento padrão, representados pela combinação de trifloxistrobina e tebuconazole com oxicloreto de cobre.

Palavras-chave: Citrus reticulata, Alternaria alternata, controle químico

\section{ABSTRACT}

Nouhra, G.; Poloni, N.M.; Pereira, F.D.; Goes, A. Efficiency of trifloxystrobin and tebuconazole, in a commercial formulation, associated with protective fungicides to control Alternaria brown spot on 'Murcott' tangors. Summa Phytopathologica, v.47, n.2, p.122-125, 2021.

Among fungal diseases, Alternaria brown spot (ABS) is the most important disease for "Murcott" tangors, causing leaf drop, spots, fruit drop and consequently heavy losses. The present study evaluated the efficiency of two levels of trifloxystrobin and tebuconazole, in commercial formulation, combined with pyrimethanil or water-soluble copper, in comparison with copper oxychloride, on ABS control in a 15-year-cultivation "Murcott" tangor orchard in Estiva Gerbi, Sao Paulo State, Brazil. Treatments were composed of the tank mixture of trifloxystrobin and tebuconazole at $0.80 \mathrm{~L} \mathrm{ha}^{-1}$ with the following fungicides ( $\mathrm{L}$ or kg commercial product $\left.2000 \mathrm{~L}^{-1}\right)$ : T1 - pyrimethanil (1.0); T2 pyrimethanil (2.0); T3 - soluble copper sulfur (1.0); T4 - copper oxychloride (3.60). Split-block design was adopted, in subplots, with three replicates containing ten plants on the row between two adjacent rows. Fungicides were applied with an FM Copling turbo sprayer, at a flow of $100 \mathrm{~mL}\left(\mathrm{~m}^{3}\right)^{-1}$. In total 17 fungicide applications were performed at intervals varying between 12 and 15 days. Evaluations consisted of determining the severity levels of symptoms on fruits in three periods (S1, S2 and S3) and the number of injuries on fruits at harvest. Treatments combining trifloxystrobin and tebuconazole with pyrimethanil, in two levels, were efficient in controlling the pathogen, similarly to the results of the standard treatment, represented by the combination of trifloxystrobin and tebuconazole with copper oxychloride.

Keywords: Citrus reticulata, Alternaria alternata, chemical control

A citricultura é de suma importância ao Brasil, cuja produção foi de 398,35 milhões de caixas $(40,8 \mathrm{~kg}$ ) na safra 2017/18 e de 285,98 milhões de caixas $(40,8 \mathrm{~kg})$ para a safra $2018 / 19(13,17)$. O Estado de São Paulo destaca-se como o maior produtor de citros, no Brasil, cuja produção para a safra 2019/20 é estimada em 388,42 milhões de caixas. Neste estado, cerca de $53 \%$ da produção é destinada ao processamento industrial, o que faz do Brasil o maior produtor mundial (17).

De acordo com dados do Departamento de Agricultura dos Estados Unidos - USDA (20) foram produzidas 53,7 milhões de toneladas de laranja na safra 2016/17, contra 30,2 milhões de tangerina e 7,6 milhões de limão e lima $(20,22)$. No Brasil, para o mesmo ano a área ocupada com laranja no país foi de 669,195 hectares, 49,281 ha para tangerina e 47,609 ha com limão $(14,22)$.

O cultivo da tangerina no Brasil está concentrado no Sul e Sudeste do País. Rio Grande do Sul, São Paulo, Paraná e Minas Gerais respondem por cerca de $80 \%$ da área cultivada com a cultura no Brasil e por $87 \%$ da produção $(14,22)$.

A cultura dos citros é afetada por diversos problemas de ordem fitossanitária, com destaque para a doença causada pela bactéria Candidatus Liberibacter asiaticus e $C a$. Li americana, responsáveis 
pelo HLB (huanglongbing) ou Greening, ), uma doença devastadora em diversas partes do mundo (7), e a mancha marrom de Alternária (MMA), causada por Alternaria alternata $(5 ; 15)$.

A mancha marrom de Alternária (MMA), é responsável por elevados prejuízos ems pomelos, tangerinas e tangores. Praticamente todas as espécies que contenham Citrus reticulata dentre os progenitores são suscetíveis ao patógeno (18).

A MMA infecta tecidos jovens, causando desfolha, seca de ramos, queda de frutos jovens e redução da produção $(2,9)$. O controle da doença deve ser realizado mediante o uso de fungicidas protetores constituídos pelos cúpricos, e por estrobilurina e triazóis (1).

O objetivo do presente trabalho foi avaliar a eficiência de trifloxistrobina e tebuconazol em mistura de tanque com pirimetanil, em duas dosagens, ou sulfato de cobre, comparado com a mistura contendo oxicloreto de cobre, no controle da MMA, sob condições de campo, em Estiva Gerbi/SP.

\section{MATERIAL E MÉTODOS}

O experimento foi realizado em propriedade particular, na safra 2017/18, em pomar de tangor 'Murcott', com plantas de 15 anos de idade, implantadas no espaçamento de 7,0 x 3,0 m, no município de Estiva Gerbi (SP). As coordenadas geográficas do local são latitude $22^{\circ}$ $40^{\prime} 35^{\prime \prime}$ 'S e longitude $49^{\circ} 28^{\prime} 8^{\prime \prime} \mathrm{O}$. Os indicadores locais são de elevada incidência e severidade de sintomas da MMA. O manejo do pomar consistiu na adoção de adubação via solo e foliar, e uso de roçadeira ecológica para controle de plantas daninhas.

O delineamento experimental utilizado foi o de Split-block, em subparcelas com três repetições contendo dez plantas na linha central. Cada unidade amostral foi representada por três linhas de plantio, adjacentes entre si, com um total de 30 plantas. As linhas externas constituíram-se em bordaduras.

Os tratamentos constituíram-se de Nativo ${ }^{(}$(Bayer SA, tebuconazol $200 \mathrm{~g} \mathrm{~L}^{-1}+$ trifloxistrobina $100 \mathrm{~g} \mathrm{~L}^{-1}$, suspensão concentrada) associado a Mythos ${ }^{\circledR}$ (Bayer SA, São Paulo, pirimetanil $300 \mathrm{~g} \mathrm{~L}^{-1}$, suspensão concentrada), Bayfolan ${ }^{\circledR}$ [Bayer SA, São Paulo, cobre (solúvel em água) $5 \%\left(61,5 \mathrm{~g} \mathrm{~L}^{-1}\right)$, carbono orgânico total 10,0\% $\left(123,0 \mathrm{~g} \mathrm{~L}^{-1}\right)$, Nitrogênio (solúvel em água) 3,0\% (36,9 $\left.\mathrm{g} \mathrm{L}^{-1}\right)$ ] e Recop (Aubaugh Agro Brasil Ltda, $840 \mathrm{~g} \mathrm{~kg}^{-1}$, ou o correspondente a $500 \mathrm{~g}$ de cobre metálico, WP).

A aplicação dos fungicidas deu-se mediante turbopulverizador FM Copling, com capacidade para 4.000L. Foi adotado volume de calda de cerca de $100 \mathrm{~mL}\left(\mathrm{~m}^{3}\right)^{-1}$. Os tratamentos avaliados foram os seguintes: T1- 0,8L trifloxistrobina + tebuconazole $+1,0 \mathrm{~L}$ de pirimetanil; T2$0,8 \mathrm{~L}$ trifloxistrobina + tebuconazole $+1,5 \mathrm{~L}$ de pirimetanil; $\mathrm{T} 3-0,8 \mathrm{~L}$ trifloxistrobina + tebuconazole $+1,0 \mathrm{~L}$ de cobre solúvel em água a $5 \%$, em formulação comercial acrescida de N; T4- 0,8L trifloxistrobina + tebuconazole $+3,6 \mathrm{~kg}$ de oxicloreto de cobre. Em todos os tratamentos foi acrescido óleo vegetal Veget Oilß a 0,25\% (v/v). Devido aos riscos potenciais da disseminação autóctone de conídios de A. alternata, agente causal da MMA, o experimento não contou com testemunha.

No total foram realizadas 17 aplicações de fungicidas, iniciadas em agosto/2016 e terminadas em maio/2017. O intervalo entre aplicações dos fungicidas variou de 12 a 15 dias.

As avaliações consistiram na determinação de: (i) severidade de sintomas nos frutos, empregando-se escala de notas, sendo: 0 - ausência de sintomas; 1-1 a 3 lesões; 2- 4 a 10 lesões e; 3- mais que 10 lesões por fruto e; (ii) determinação dos níveis de cicatrizes nos frutos, quando da colheita. Para essa variável foi empregada escala de notas semelhante à descrita anteriormente, porém, em vez do número de lesões, levou-se em conta o número de cicatrizes. Os dados obtidos foram avaliados mediante análise de variância, sendo empregado o Programa AgroEstat (4). As médias obtidas foram comparadas pelo teste de Tukey com 5\% de probabilidade.

\section{RESULTADOS E DISCUSSÃO}

Nas duas primeiras avaliações, correspondentes a S1 e S2 (Tabela 1), constatou-se que a mistura de trifloxistrobina + tebuconazole associado a 1,0 ou $1,5 \mathrm{~L}$ de pirimetanil foram superiores ao tratamento constituído pela combinação de trifloxistrobina + tebuconazole a sulfato de cobre ou oxicloreto de cobre. Na terceira avaliação, correspondente a S3, constatou-se que estatisticamente não houve diferença entre os tratamentos constituídos por trifloxistrobina + tebuconazole associado a 1,0 L pirimetanil, 1,0L de cobre solúvel ou 3,6 kg de oxicloreto de cobre (Tabela 1).

Em relação ao número de cicatrizes nos frutos, constatou-se que a melhor resposta foi obtida mediante a combinação de trifloxistrobina + tebuconazole e pirimetanil a 1,5 L.

A MMA é a doença fúngica mais importante das tangerineiras no Brasil, onde são empregadas anualmente cerca de 15 aplicações de fungicidas (19). Junto ao Ministério da Agricultura, especificamente para o controle da MMA, encontram-se registrados apenas os fungicidas Nativo e Recop, os quais correspondem a formulações comerciais constituídas respectivamente por trifloxistrobina e tebuconazole e oxicloreto de cobre (http://agrofit.agricultura.gov.br/agrofit/cons/ principal_agrofit_cons). Entretanto, para as demais doenças fúngicas associadas aos citros, especialmente a pinta preta, causada por Phyllosticta citricarpa e podridão floral, causada por Colletotrichum gloeosporioides e C. abscissum, e verrugose das laranjas doces e limões, causadas por Elsinoe australis e E. fawcettii, encontram-se registrados fungicidas dos grupos das estrobilurinas, inibidores da desmetilação e fungicidas cúpricos, de diversas fontes $(8,12)$. Dessa feita, pela extensão de uso aplicada, fungicidas destes grupos químicos, e prescritos na Lista PIC, tornam-se rotineiramente utilizados na cultura, o que permite a flexibilização das alternativas de produtos químicos utilizados (12).

Tabela 1. Eficiência de trifloxistrobina e tebuconazole, em formulação comercial, associados a fungicidas protetores no controle da mancha marrom de alternaria em tangor "Murcott". Estiva Gerbi, 2017.

\begin{tabular}{ccccc}
\hline Tratamentos & \multicolumn{3}{c}{ Severidade } & Cicatrizes \\
\hline & $\mathrm{S} 1$ & $\mathrm{~S} 2$ & $\mathrm{~S} 3$ & \\
\hline $\mathrm{T} 1$ & $0,26 \mathrm{c}$ & $0,51 \mathrm{bc}$ & $0,91 \mathrm{ab}$ & $1,62 \mathrm{~b}$ \\
$\mathrm{~T} 2$ & $0,18 \mathrm{c}$ & $0,46 \mathrm{c}$ & $0,67 \mathrm{~b}$ & $1,34 \mathrm{c}$ \\
$\mathrm{T} 3$ & $0,61 \mathrm{~b}$ & $0,84 \mathrm{~b}$ & $1,14 \mathrm{ab}$ & $1,74 \mathrm{ab}$ \\
$\mathrm{T} 4$ & $1,33 \mathrm{a}$ & $1,19 \mathrm{a}$ & $1,32 \mathrm{a}$ & $2,18 \mathrm{a}$
\end{tabular}

Tratamentos: T1- 0,8L de trifloxistrobina + tebuconazole $+1,0 \mathrm{~L}$ de pirimetanil; T2- $0,8 \mathrm{~L}$ de trifloxistrobina + tebuconazole $+1,5 \mathrm{~L}$ de pirimetanil; $\mathrm{T} 3-0,8 \mathrm{~L}$ trifloxistrobina + tebuconazole $+1,0 \mathrm{~L}$ de cobre solúvel em água a $5 \%$, em formulação comercial acrescida de N; T4- $0,8 \mathrm{~L}$ de trifloxistrobina + tebuconazole $+3,6 \mathrm{~kg}$ de oxicloreto de cobre. S1- Severidade dos sintomas de MMA em frutos de tangor 'Murcott', em 23/03/2017; S2 - Severidade dos sintomas de MMA em frutos de tangor 'Murcott', em 05/07/2017; S3 - Severidade dos sintomas de MMA em frutos de tangor 'Murcott', em 18/07/2017. Cicatrizes - Notas do número de cicatrizes em frutos de tangor 'Murcott', em avaliação realizada em 18/07/2017. 
Comparativamente, do ponto de vista estatístico não foi constatada diferença entre as combinações contento trifloxistrobina + tebuconazole e pirimetanil a 1,0 e 1,5 L. Entretanto, em termos absolutos, os níveis de severidade dos sintomas no tratamento contendo $1,5 \mathrm{~L}$ de pirimetanil foi o que resultou em menor quantidade de sintomas de MMA, independente das avaliações.

Os fungicidas mais utilizados para o controle da MMA, no Estado de São Paulo, são a piraclostrobina, isoladamente ou em combinação com oxicloreto de cobre ou hidróxido de cobre. Entretanto, mesmo com o elevado número de pulverizações sequenciais, em muitas áreas de produção o controle da doença mostra-se aquém do desejável.

No Estado de São Paulo, há diversos fatores associados aos baixos níveis de controle de MMA. Dentre os fatores associados incluem-se o elevado nível de suscetibilidade dos materiais genéticos, condições altamente predisponentes ao patógeno, como por exemplo, ambiente favorável e fluxos vegetativos recorrentes, intervalos inadequados entre as aplicações dos fungicidas, falha na tecnologia de aplicação, dentre outros. A possibilidade da redução da sensibilidade do agente causal às estrobilurinas também é especulada.

Nos últimos anos, diversos estudos têm demonstrado a existência de A. alternata resistente às estrobilurinas. Um dos primeiros trabalhos publicado relata a ocorrência de isolados resistentes no Estado da Flórida, nos Estados Unidos da América, em 2005 (16). Posteriormente, em estudo realizado com 817 isolados obtidos de pomares cítricos, neste mesmo Estado, Vega \& Dewdney (21) constataram que 57,6\% mostraram-se resistentes a azoxistrobina e piraclostrobina. No Brasil, resultados convergentes foram também publicados por Condé (11), com isolados de diversas partes do Brasil, e Chitolina et al. (10), em isolados originários de plantas e frutos sintomáticos, coletados no estado São Paulo, na safra de 2017. Os resultados convergem com informações recentes obtidas por Baraldi (3), mediante isolados de $A$. alternata obtidos de $C$. reticulata e seus híbridos, obtidos nos Estados de São Paulo, Minas Gerais e Rio de Janeiro (3). O significado prático do comportamento do fungo, conforme apontado, ainda não foi estabelecido, mas certamente o agravamento nos níveis de controle de MMA é esperado.

Pirimetanil é um fungicida de contato do grupo químico anilopirimidina. Em citros, no Brasil, este fungicida é registrado para o controle de $P$. citricarpa, em pulverizações convencionais, e também para o controle de diversas doenças de plantas, nas fases de florescimento e na pré-colheita. No presente estudo, este fungicida mostrou potencial para o controle de A. alternata em tangor "Murcott', quando associado a trifloxistrobina e tebuconazole. De acordo com os resultados obtidos neste estudo, a eficiência de controle de A. alternata, em tangor "Murcott' foi pelo menos comparável aos resultados obtidos mediante ao emprego de oxicloreto de cobre. Estudos complementares, em diversas condições ambientais, visando à convalidação dos resultados, fazem-se necessários. Além do exposto, em citros, o uso recorrente de fungicidas cúpricos, em determinadas condições, pode resultar em danos na casca dos frutos, o que é indesejável para o mercado de frutas frescas $(6,19,20)$. No presente estudo, embora não dimensionado, os frutos tratados com pirimetanil, independente da dosagem avaliada exibiam excelente aparência, com possível indicação desta característica adicional.

O emprego simultâneo de trifloxistrobina e tebuconazole, na formulação comercial a $0,80 \mathrm{~L}$ associado a pirimetanil a $1,5 \mathrm{~L}$ por $2.000 \mathrm{~L}$ de água, em pulverização convencional, na vazão de $100 \mathrm{~mL}\left(\mathrm{~m}^{3}\right)^{-1}$ é eficiente no controle da MMA em tangor 'Murcott'. Por tratar-se de resultado advindo de um único experimento, a convalidação deste estudo, sob condições ambientais diversas, faz-se necessário.

\section{REFERÊNCIAS}

1. Azevedo, F.A.; Martelli, I.B.; Polydoro, D.A.; Pacheco, C.D.A.; Schinor, E.H.; Bastianel, M. Positive relationship between citrus leaf miner and alternaria brown spot. Ciência Rural, Santa Maria, v.45, n.7, p.1160-1163, 2015.

2. Azevedo, F.A.; Polydoro, D.A.; Bastianel, M.; Kupper, K.C.; Stuart, R.M.; Costa, F.P.; Pio, R.M. Resposta de diferentes genótipos de tangerinas e seus híbridos à inoculação in vitro e in vivo de Alternaria alternata. Revista Brasileira de Fruticultura, Jaboticabal, v.32, n.3, p.1-10, 2010.

3. Baraldi, A.L.M. Sensibilidade de Alternaria alternata: agente causal da mancha marrom de alternária a fungicidas do grupo químico das estrobilurinas. 2019. 18f. Trabalho de Conclusão de Curso (Graduação em Engenharia Agronômica) - Faculdade de Ciências Agrárias e Veterinárias, Universidade Estadual Paulista, Jaboticabal.

4. Barbosa, J. C.; Maldonado Júnior, W. Software AgroEstat: Sistema de análises estatísticas de ensaios agronômicos. Universidade Estadual Paulista, Faculdade de Ciências Agrárias e Veterinárias, Jaboticabal, 2009.

5. Bassanezi, R.B.; Silva Jr, G.J.; Feichtenberger, E.; Belasque Jr, J.; Behlau, F.; Wulff, N.A. Manual de fitopatologia. In: Amorim, L.; Rezende, J.A.M.; Bergamin Filho, A.; Camargo, L.E.A. (Eds.) Manual de Fitopatologia: Doenças das Plantas Cultivadas. 5.Ed. Ouro Fino: Editora Agronômica Ceres, v.2, p.271-306, 2016.

6. Bhatia, A; Roberts, P.; Timmer, L.W. Evaluation of the Alter-Rater Model for Timing of Fungicide Applications for Control of Alternaria Brown Spot of Citrus. Plant Disease, St Paul, v.87, n.9, p.1089-1093, 2003.

7. Bové, J.M. Huanglongbing: a destructive, newly-emerging, century-old disease of citrus. Journal Plant Pathology, Bary, v.88, n.1, p.7-37, 2006.

8. Brasil. Ministério da Agricultura, Pecuária e Abastecimento. AGROFIT: Sistemas de agrotóxicos fitossanitários. Brasília, DF: MAPA, 2019. Disponível em: http://extranet.agricultura.gov.br/agrofit_cons/principal_agrofit_cons $>$. Acesso em 03 out. 2019.

9. Carvalho, D.D.C.; Alves, E.; Camargos, R.B.; Oliveira, D.F.; Scolforo, J.R.S.; Carvalho; D.A. Plant extracts to control Alternaria alternata in Murcott tangor fruits. Revista Iberoamericana de Micología, Barcelona, v.28 p.173-178, 2011.

10. Chitolina, G.M.; Silva-Junior, G.J.; Feichtenberger, E.; Pereira, R.G.; Amorim, L. First report on quinone outside inhibitor resistance of Alternaria alternata causing Alternaria brown spot in tangerines in São Paulo, Brazil. Plant Health Progress, St. Paul, v.20, n.2, p.94, 2019.

11. Condé, T.O. Caracterização da resistência de isolados de Alternaria alternata patótipo tangerina a estrobilurinas. 2018. 52f. Dissertação (Mestrado em Microbiologia Agrícola) - Universidade Federal de Lavras, Lavras.

12. Fundecitrus. Grade de inseticidas, acaricidas, fungicidas da produção integrada dos citros - PIC Brasil. Araraquara, SP: Fundecitrus, 2018. Disponível em: <https://www.fundecitrus.com.br/listapic $>$. Acesso em: 01 out. 2019.

13. Fundecitrus. Revista Citricultor. Sucesso do Manejo Externo - Edição 50. Araraquara, v.1, p.10, 2019. Disponível em: < https://www.fundecitrus. com.br/comunicacao/revista detalhes/revista- citricultor---edicao-50/61 >. Acesso em: 21 nov. 2019.

14. IBGE. Censo Agropecuário 2018. Rio de Janiero, RJ: IBGE, 2017. Disponível em: ‘https://sidra.ibge.gov.br/pesquisa/censo-agropecuario/ censo-agropecuario-2017>. Acesso em: 28 out. 2019.

15. Kiely, T.B. Brown spot of Emperor mandarin. Agricultural Gazette of new South Wales, Sidney, v.75, n.2, p.854-856, 1964

16. Mondal, S.N.; Bhatia, A.; Shilts, T.; Timmer, L.W. Baseline Sensitivities of Fungal Pathogens of Fruit and Foliage of Citrus to Azoxystrobin, Pyraclostrobin, and Fenbuconazole. Plant Disease, Saint Paul, v.89, n.11, p.1186-1194, 2005.

17. Fundecitrus. Pesquisa de Estimativa de Safra: Reestimativa da safra de laranja 2019/2020 do Cinturão Agrícola de São Paulo e Triângulo/Sudoeste Mineiro - Cenário em Setembro 2019. Araraquara, SP: Fundecitrus, 2019. Disponível em: $<$ https://www.fundecitrus.com.br/pdf/pes_relatorios/0919 Reestimativa_da_safra_de_laranja2.pdf $>$. Acesso em: 20 nov. 2019.

18. Reis, R.F.; Almeida, T.F.; Stuchi, E.S.; Goes, A. Suspeptibility of citrus species to Alternaria alternata, the causal agent of Alternaria brown spot. Scientia Horticulturae, Amsterdam, v.113, p.336-342, 2007.

19. Timmer, L.W.; Peever, T.L.; Solel, Z.; Azuya, K. Alternaria disease of citrus-novel pathosystems. Phytopathologia Mediterranea, Bologna, v.42, n.2, p.99-112, 2003 
20. Timmer, L.W.; Soleil, Z.; Orozco-Santos, M. Alternaria Brown Spot Of Mandarines. In: Timmer, L.H.; Garnsey, S.M.; Graham, J. H. (ed.). Compendium of Citrus Diseases. 2.ed. Minnesota: APS Press Saint Paul, 2000. v.1, p.19.

21. USDA. Citrus: world markets and trade. Cidade: USDA, 2018. Disponível em: <https://www.fas.usda.gov/data/citrus-world-markets- and-trade>. Acesso em: 14 out. 2019.
22. Vega, B.; Dewdney, M.M. Distribution of QoI resistance in populations of tangerine-infecting Alternaria alternata in Flórida. Plant Disease, Saint Paul, v.98, n.1, p.67-76, 2014.

23. Vidal, M.F. Citricultura na área de atuação no BNB 2018. Caderno Setorial Escritório Técnico de Estudos Econômicos do Nordeste, Fortaleza, CE, ano 3, n.41, 2018. Disponível em: <https://www.bnb.gov.br/docu4 ments/80223/404948 0/41_Citrus_2018.pdf/11e22002-730c-a18b-0d0fO $-479 f 9 \mathrm{e} 4 \mathrm{~d} 3309>$. Acesso em 21 nov. 2019. 